\title{
A short note on Dynkin groups and convergence groups
}

\author{
Aslı Yaman
}

\begin{abstract}
We prove that the family of discrete non-elementary Dynkin groups coincides with the family of non-elementary convergence groups.
\end{abstract}

Mathematics Subject Classification (2000). 20F38, 20 F65.

Keywords. Dynkin groups, convergence groups.

\section{Introduction}

Convergence groups have a prominent role in the study of geometric groups. They were introduced by Gehring and Martin in [GM] in order to describe the dynamical properties of Kleinian groups, i.e. discrete subgroups of isometries of the hyperbolic $n$-space $\mathbb{H}^{n+1}$, acting on the boundary of $\mathbb{H}^{n+1}$. The notion of convergence group was later on generalised to actions on compact Hausdorff spaces by several people, such as Tukia, Freden and Bowditch (see [T], [Fr], [B]). The groups acting properly discontinuously on a complete locally compact Gromov hyperbolic space are the motivation examples for the general case, hence include in particular hyperbolic groups. It is well known that such groups induce a convergence action on the boundary of the Gromov hyperbolic space.

Grigorchuk asked whether convergence groups and Dynkin groups might be related since they admit similar kind of dynamics. Indeed this note answers this question and shows the following.

Theorem. The family of discrete "non-elementary" Dynkin groups coincides with the family of "non-elementary" convergence groups.

Dynkin groups and spaces were defined by Furstenberg in order to study boundaries of finitely generated groups. In particular Dynkin groups and Dynkin sets (see [Fu] for the definition) give a natural generalisation of hyperbolic groups and their boundaries. Recall that the boundary of a hyperbolic group is the boundary of a complete locally compact Gromov hyperbolic space on which the hyperbolic group acts properly discontinuously and cocompactly. Hence the theorem shows that 
convergence groups and Dynkin groups induce the same generalisation for "nonelementary" cases.

I would like to thank to R. Grigorchuk for bringing the question to my attention and to the referee for his comments.

\section{Definitions and results}

Let us recall some definitions (see also [Fu]).

Definition 1. Let $\Gamma$ be a locally compact topological group and $M$ be a compact metrizable space on which $\Gamma$ acts by homeomorphisms. The space $M$ is a Dynkin space of $\Gamma$ if, for every $\varepsilon>0$, there is a compact subset $F_{\varepsilon}$ of $\Gamma$ such that, for each $\gamma \in \Gamma \backslash F_{\varepsilon}$, there exist $x, y \in M$ such that $\gamma$ sends the complement of an $\varepsilon$-neighborhood of $x$ into an $\varepsilon$-neighborhood of $y$.

Definition 2. A group $\Gamma$ is a Dynkin group if it possesses a Dynkin space $M$ with no global fixed point.

In this note we are interested only in discrete groups. Therefore each Dynkin group is assumed to be discrete, hence in its definition the compact set $F_{\varepsilon}$ is always a finite set. The following lemma establishes a dichotomy between "elementary" and "non-elementary Dynkin groups" similar to "elementary" and "non-elementary convergence groups".

Lemma. Let $\Gamma$ be a Dynkin group and $(M, d)$ be a Dynkin space of $\Gamma$ with its metric $d$. Then either $\Gamma$ preserves setwise two points of $M$ or each orbit in $M$ has infinite cardinality; in particular it is infinite.

We shall thus refer to Dynkin groups preserving setwise two points of $M$ as elementary Dynkin groups.

Proof. Suppose that $\mathrm{O}=\left\{x_{1}, \ldots, x_{n}\right\}$ is a finite orbit of $\Gamma$ in $M$ with $n \geq 3$. Then clearly the subgroup $H=\left\{\gamma \mid \gamma x_{i}=x_{i}\right\}$ is infinite.

Let $4 \varepsilon<\min \left\{d\left(x_{i}, x_{j}\right), i e q j\right\}$. Since $\Gamma$ is a Dynkin group there exists $F_{\varepsilon}$ such that for all $\gamma \notin F_{\varepsilon}$ there are $x, y \in M$ so that the complement of an $\varepsilon$-neighbourhood $N_{\varepsilon}(x)$ is sent by $\gamma$ into an $\varepsilon$-neighbourhood $N_{\varepsilon}(y)$ of $y$.

Let $\gamma_{n}$ be an infinite sequence of distinct elements of $H \backslash F_{\varepsilon}$. Then for all $n$ there are $a_{n}, b_{n} \in M$ such that for all $z \notin N_{\varepsilon}(x)$ we have $\gamma_{n} z \in N_{\varepsilon}(y)$. As $M$ is compact, after passing to a subsequence $a_{n}$ and $b_{n}$ converge respectively to $a$ and $b$, and so, for $n$ large enough, $d\left(a_{n}, a\right) \leq \varepsilon$ and $d\left(b_{n}, b\right) \leq \varepsilon$. Since $\mathrm{O}$ has at least three elements and $d\left(x_{i}, x_{j}\right)>4 \varepsilon$, there exists $i \neq j$ with $x_{i}, x_{j} \notin N_{2 \varepsilon}(a)$. Hence $x_{i}, x_{j} \notin N_{\varepsilon}\left(a_{n}\right)$ 
for $n$ large enough, which implies that $\gamma_{n} x_{i}$ and $\gamma_{n} x_{j}$ are in $N_{\varepsilon}\left(b_{n}\right) \subset N_{2 \varepsilon}(b)$ for large enough $n$. This is a contradiction since $\gamma_{n} x_{i}=x_{i}$ for all $i$ and so $D\left(\gamma x_{i}, \gamma x_{j}\right)>4 \varepsilon$ by the choice of $\varepsilon$. This shows that there are no orbits of cardinality that is finite and greater than 2 in $M$.

We now give the definition of a convergence group. We mention that the notion of convergence group can be defined in greater generality for the non-discrete case (See $[\mathrm{T}])$. We restrict ourself to the discrete case.

Definition 3. Let $\Gamma$ be a group acting on compact metrizable space $M$ by homeomorphisms. The group $\Gamma$ is a convergence group if, for any sequence $\left\{\gamma_{i}\right\}_{i}$ of distinct elements of $\Gamma$, there are two points $a, b \in M$ and a subsequence $\left\{\gamma_{i_{n}}\right\}_{n}$ such that $\left\{\gamma_{i_{n}}\right\}_{n}$ converges to $b$ locally uniformly on $M \backslash\{a\}$ as $n$ tends to $\infty$.

A convergence group is elementary if it is finite or preserves setwise a nonempty subset with at most 2 elements of $M$. As an analogue of the lemma above we know that a non-elementary convergence group does not fix any finite set in $M$. When $M$ has at least 3 points, Definition 3 is equivalently formulated in [B] as follows.

Definition 4. Let $\Gamma$ be a group acting on a compact metrizable space $M$ by homeomorphisms and let $M$ have at least 3 points. The group $\Gamma$ is a convergence group if its action on the space of distinct triples, $\Theta_{3}(M)$, is properly discontinuous (i.e., for any compact subset $K \subseteq \Theta_{3}(M)$ the set $\{\gamma \in \Gamma \mid \gamma K \cap K \neq \emptyset\}$ is finite).

We note that the first definition gives a dynamical characterization of convergence groups, while the second one is more natural topologically, given that $\Theta_{3}(M)$ can be compactified by adding a copy of $M$. This compactification can be described by presenting $\Theta_{3}(M) \sqcup M$ as a quotient of $M \times M \times M$, where the quotient map is the identity on $\Theta_{3}(M)$ and sends a triple $(x, y, z)$ to $a \in M$ if at least two of $x$, $y, z$ are equal to $a$. We will refer to the topology thus defined on $\Theta_{3}(M) \sqcup M$ as the topology of compactification. In this topology we can see that if $\left(x_{i}, y_{i}, z_{i}\right)_{i}$ is a sequence in $\Theta_{3}(M)$ with $x_{i}$ and $y_{i}$ tending to $a$, then $\left(x_{i}, y_{i}, z_{i}\right)$ converges to $a$ in $\Theta_{3}(M) \sqcup M$. The converse is also true up to permuting $x_{i}, y_{i}, z_{i}$. Therefore the action of a convergence group $\Gamma$ can be extended to $\Theta_{3}(M)$ with $(x, y, z) \mapsto(\gamma x, \gamma y, \gamma z)$ for every $\gamma \in \Gamma$.

Proof of Theorem. We first prove that if $\Gamma$ is a non-elementary Dynkin group then it is a non-elementary convergence group.

Let $\Gamma$ be a non-elementary Dynkin group and $(M, d)$ be a Dynkin space. Thus $M$ has at least 3 elements. Let us assume that $\Gamma$ is not a convergence group. Thus $\Gamma$ does not act properly discontinuously on $\Theta_{3}(M)$. Hence there exist an infinite sequence $\left\{\gamma_{n}\right\}_{n}$ of distinct elements in $\Gamma$ and $\left(a_{n}, b_{n}, c_{n}\right) \in \Theta_{3}(M)$ such that $\left(a_{n}, b_{n}, c_{n}\right) \rightarrow$ $(a, b, c) \in \Theta_{3}(M)$ and $\gamma_{n}\left(a_{n}, b_{n}, c_{n}\right) \rightarrow(x, y, z) \in \Theta_{3}(M)$. 
Choose $\varepsilon$ such that $6 \varepsilon<\min \{d(a, b), d(b, c), d(a, c), d(x, y), d(y, z), d(x, z)\}$. Thus there exists a finite set $F_{\varepsilon}$ in $\Gamma$ such that for all $\gamma \in \Gamma \backslash F_{\varepsilon}$ there exist $u, v \in M$ such that the complement of an $\varepsilon$-neighborhood $N_{\varepsilon}(x)$ of $x$ is sent by $\gamma$ into an $\varepsilon$ neighborhood $N_{\varepsilon}(y)$ of $y$. Therefore, without loss of generality, we can assume that, for all $n$, there exist points $u_{n}, v_{n}$ in $M$ with $\gamma_{n}\left(M \backslash N_{\varepsilon}\left(u_{n}\right)\right) \subset N_{\varepsilon}\left(v_{n}\right)$. Since $M$ is a compact space we know that, after passing to subsequences of $\left\{u_{n}\right\}_{n}$ and $\left\{v_{n}\right\}_{n}, u_{n}$ and $v_{n}$ converge to $u, v$ in $M$, respectively. For $n$ large enough, we have $d\left(u_{n}, u\right) \leq \varepsilon$, $d\left(v_{n}, v\right) \leq \varepsilon$. By the choice of $\varepsilon$ we can assume, without loss of generality, that for all $n$, we have $a, b \notin N_{3 \varepsilon}(u)$ and hence $a_{n}, b_{n} \notin N_{\varepsilon}\left(u_{n}\right)$ for all large $n$. That implies that, for all large $n, \gamma_{n} a_{n}$ and $\gamma_{n} b_{n}$ are in $N_{\varepsilon}\left(v_{n}\right)$, hence $d(x, y) \leq \varepsilon$. This is in contradiction with the choice of $\varepsilon$.

For the other direction we prove that every convergence group is a Dynkin group.

Let $\Gamma$ be a convergence group acting on a compact metrizable space $(M, d)$ where $d$ denotes the metric on $M$.

Assume that $M$ is not a Dynkin space for $\Gamma$. Then there exist an $\varepsilon$ and an infinite sequence $\left\{\gamma_{n}\right\}_{n}$ in $\Gamma$ such that for all $x, y$ in $M$ and for all $n$ there exists a point $z_{n} \in M \backslash N_{\varepsilon}(x)$ with $\gamma_{n} z_{n} \notin N_{\varepsilon}(y)$. As $\Gamma$ is a convergence group, after passing to a subsequence we see that there are two points $a, b \in M$ such that $\gamma_{n}$ converges to $b$ locally uniformly on $M \backslash\{a\}$. Now, by hypothesis, for all $n$, there exists $z_{n} \in M \backslash N_{\varepsilon}(a)$ with $\gamma_{n} z_{n} \in M \backslash N_{\varepsilon}(b)$, hence $\gamma_{n} z_{n}$ converges to $z \in M \backslash N_{\varepsilon}(b)$. This is a contradiction since $\gamma_{n} z_{n}$ converges to $b$ by locally uniform convergence on $M \backslash\{a\}$ of $\gamma_{n}$.

\section{References}

[B] B. H. Bowditch, Convergence groups and configuration spaces. In Group theory down under (Canberra, 1996), Walter de Gruyter, Berlin 1999, 23-54. Zbl 0952.20032 MR 1714838

[Fr] E. M. Freden, Properties of convergence groups and spaces. Conform. Geom. Dyn. 1 (1997), 13-23. Zbl 0983.57029 MR 1452414

[Fu] H. Furstenberg, Poisson boundaries and envelopes of discrete groups. Bull. Amer. Math. Soc. 73 (1967), 350-356. Zbl 0184.33105 MR 0210812

[GM] F. W. Gehring and G. J. Martin, Discrete quasiconformal groups. I. Proc. London Math. Soc. (3) 55 (1987), 331-358. Zbl 0628.30027 MR 0896224

[T] P. Tukia, Convergence groups and Gromov's metric hyperbolic spaces. New Zealand J. Math. 23 (1994), 157-187; erratum ibid. 25 (1996), 105-106. Zbl 0855.30036 MR 1313451

Received April 1, 2006; revised May 2, 2006

Centre de Recerca Matemàtica, Apartat 50, 08193, Bellaterra, Spain

E-mail: ayaman@crm.es 